\section{deCode-ing autism}

\section{By Lev Osherovich, Senior Writer}

Recent general media coverage has suggested a gene sequencing study by deCode genetics ehf showed a clear link between paternal age and mutations that might cause autism spectrum disorder and schizophrenia. However, the newspapers failed to note that a direct relationship between the mutations described in the paper and autism spectrum disorder or schizophrenia remains to be established. The papers also glossed over the main finding of the study-there is a paternal origin for genetic variation in the general human population.

The deCode-led team used high throughput gene sequencing to identify new mutations in the genomes of a handful of individuals that are absent in their parents and thus are likely to have spontaneously arisen in the father's sperm. ${ }^{1}$ Their analysis revealed an intriguing correlation between number of mutations and father's age.

The general media described the findings as a breakthrough in understanding the roots of autism spectrum disorder (ASD) and schizophrenia but missed the true value of the study as a snapshot of human evolution in action.

"This paper sheds much less light on the pathogenesis of schizophrenia and autism than the lay press has indicated," said the study's coauthor, deCode CEO Kári Stefánsson. "This is mainly a study of nucleotide mutations in human populations."

The study quantifies the appearance of new mutations from one generation to the next and suggests that these mutations could contribute to ASD and schizophrenia, two neuropsychiatric disorders thought to have a strong genetic underpinning.

However, much more work is needed to prove that the mutations identified by the deCode team really cause neuropsychiatric disease in a broader patient population.

Answering how much these mutations really contribute to ASD and schizophrenia risk will require analysis of a much larger cohort of patients and their families.

\section{Shown and known}

Stefánsson's team gathered complete genomic data from 78 Icelandic families typically consisting of a father, a mother and their offspring. Of the offspring, 44 were autistic and 21 had schizophrenia. Altogether the team sequenced 219 individuals.

The team found a total of 4,933 SNPs in the offspring that were absent in both parents and were thus likely to have arisen at or around conception or early embryonic development. The average individual had 63.2 such de novo or spontaneous mutations that differed from the parents' genomes.

Notably, the children of older fathers had the highest number of $d e$ novo mutations. Each year of the father's age correlated with a cumulative increase of about two mutations. Advanced maternal age did not correlate with a higher mutation count.

Stefánsson thus proposed that mutation in the sperm of older fathers accounts for the majority of new genetic variation.

"This is the first paper describing genome studies of mutations that increase with age," said Stefánsson. "If you ask what causes mutation in a population, $97 \%$ of it is explained by the age of the father."

Results were published in Nature and are not patented.

\section{Disease connection?}

The deCode study builds a solid case for a paternal origin of spontaneous genetic variation, thought to be a basic mechanism of evolution. Whether these paternally derived mutations affect ASD and schizophrenia risk remains unclear.

The idea that mutations in general contribute to ASD and schizophrenia is not new. One leading theory argues that the two conditions are caused by individually rare but collectively numerous genetic variants. These variants alter the activity of proteins involved in neurological development, leading to problems in brain connectivity and cognitive functioning.

Consistent with this genetic theory, recent studies by multiple academic teams have turned up a host of chromosomal abnormalities that are likely to underlie certain highly heritable forms of ASD. ${ }^{2}$

However, not all cases of ASD can be accounted for by such gross genetic alterations, prompting a search for more subtle genetic variants such as disease-associated SNPs and copy number variants.

Mark Daly, associate professor of medicine at Harvard Medical School and an associate member of the Broad Institute of MIT and Harvard, said ASD and schizophrenia have a very high level of heritability, "more than $50 \%$, perhaps as high as $80 \%$," and thus are likely to have a strong genetic basis.

However, the deCode study focuses on new spontaneous genetic variants that are not present in the parents and thus would have been overlooked in prior studies of heritable factors in ASD.

Daly suspects that many cases of ASD are caused by a combination of inherited and spontaneous mutations, but unraveling the relative contribution of these two types of mutations is technically challenging.

Earlier this year, teams led by Daly and others sequenced the exomes of patients with ASD and uncovered rare de novo mutations in known ASDlinked genes and new ASD candidate genes. ${ }^{3-5}$

Because numerous prior epidemiological studies have established that paternal age is a major risk factor for ASD, "it is beyond reasonable doubt that the risk of autism increases with the age of the father," said Stefánsson. "What is controversial is how large a percentage of this increase is explained" by de novo mutations such as those uncovered in the deCode study.

Stefánsson proposed that new mutations in the sperm of older fathers could be a risk factor for neuropsychiatric disease. Based on a statistical model, his team proposed that the increased mutational rate caused by high paternal age accounted for the high ASD risk that prior epidemiological studies had linked to delayed parenthood. 
Because both the age at reproduction and rates of ASD have steadily crept up in industrialized nations, he speculated that increased mutation rates could thus contribute to the rise in ASD.

"The elusive question is what has increased the rate of autism diagnosis," said Stefánsson. "I think the age of the father explains some of it but not all of it."

Daly said it is hard to extrapolate the overall impact of paternal agerelated de novo mutations on ASD risk.

"There's a lot of epidemiology on the effect of increasing age of parents on autism risk, but different studies estimate that effect at different strengths," he said.

"We know some cases of ASD arise from spontaneous point mutations. This paper shows the rate of such mutations increases with paternal age," said Daly. "While some of these mutations will without question contribute to ASD risk, what's not established is whether this increase in risk is tiny or substantial."

Daniel Geschwind, professor of neurology and psychiatry and chair of human genetics at the University of California, Los Angeles, said the sample size in Stefánsson's study was too small to draw firm conclusions about the impact of de novo mutations on ASD and schizophrenia risk.

"The fact the paternal age can account for the majority of mutation in offspring makes this paper Nature-worthy," said Geschwind. "But replicating this with a larger data set prior to publication would have been nice."

Demonstrating that paternal age leads to specific mutations in known ASD genes would help Stefánsson's case. Geschwind suggested the deCode team "narrow down the relationship between these mutations and actual autism" by identifying and characterizing individual mutations that are likely to cause ASD in the sequenced individuals.

\section{Alternative theories}

There are multiple other possibilities for why neuropsychiatric disease rises in the children of older parents.

Daly cautioned that it is unclear whether the rise in ASD diagnoses over the last few decades is due to biological mechanisms such as higher mutation rates or from social factors.

Widening of diagnostic criteria and greater awareness of the disorder may in fact contribute to the prevalence of ASD, which the Centers for Disease Control and Prevention recently estimated might affect 1 in 88 children.

"There is now a broader definition of autism that allows diagnosis in a larger number of people," said Stefánsson.

"There is lack of agreement in the field about the rate at which ASD is increasing over and above the shift in diagnosis," added Daly.

Also, greater public awareness of the disease may lead some parents to suspect an autism diagnosis, and older parents are more likely to seek medical treatment for children. ${ }^{6}$

Another possibility is that male carriers of mild hereditary forms of the disease may delay reproduction due to social difficulties in finding mates.

"Older fathers may be on the autism spectrum themselves," said Geschwind.

Stefánsson said he hopes to collaborate with Daly and other ASD researchers to test larger patient cohorts for evidence of paternally derived disease-linked mutations.

He noted that the findings are unrelated to deCode's principal commercial efforts in discovery and development of diagnostic biomarkers.

Osherovich, L. SciBX 5(34); doi:10.1038/scibx.2012.889

Published online Aug. 30, 2012

\section{REFERENCES}

1. Kong, A. et al. Nature; published online Aug. 22, 2012; doi:10.1038/nature11396

Contact: Kári Stefánsson, deCode genetics ehf, Reykjavik, Iceland e-mail: kstefans@decode.is

2. Malhotra, D. \& Sebat, J. Cell 148, 1223-1241 (2012)

3. Neale, B.M. et al. Nature 485, 242-245 (2012)

4. Sanders, S.J. et al. Nature 485, 237-241 (2012)

5. O'Roak, B.J. et al. Nature 485, 246-250 (2012)

6. Van Meter, K.C. et al. Autism Res. 3, 19-29 (2010)

COMPANIES AND INSTITUTIONS MENTIONED

Broad Institute of MIT and Harvard, Cambridge, Mass. Centers for Disease Control and Prevention, Atlanta, Ga. deCode genetics ehf, Reykjavik, Iceland Harvard Medical School, Boston, Mass. University of California, Los Angeles, Calif. 